\title{
Leiomyoma of the urinary bladder - a case report and review of literature
}

\author{
Zaid S. Khudher \\ Department of Surgery, College of Medicine, University of Mosul, Mosul, Iraq. \\ Correspondence: Zaid S. Khudher. zaidsurgeon@yahoo.com.
}

(Ann Coll Med Mosul 2018; 40 (2): 74-76).

Received: $25^{\text {th }}$ Oct. 2013; Accepted: $22^{\text {nd }}$ Jan. 2014.

\begin{abstract}
Most bladder tumors are derived from the urothelium. Benign mesenchymal tumors are rare. Leiomyoma of the bladder is the most common benign neoplasm. A case of leiomyoma of the bladder presented here with confusing finding on imaging, ultra sound and I.V. urography.
\end{abstract}

Keywords: Leiomyoma, urinary bladder.

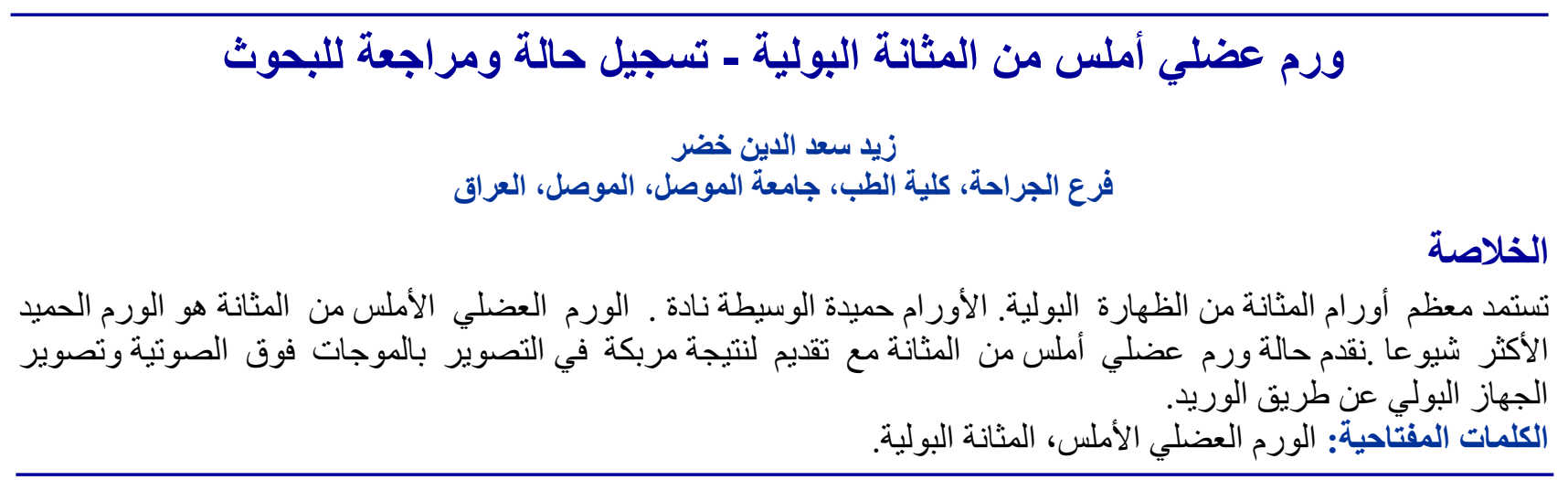

\section{INTRODUCTION}

eiomyoma of the bladder is an uncommon benign tumor ${ }^{1}$ approximately 200 cases were described in the literature ${ }^{2}$ however, it is the most frequent benign neoplasm accounting for $0.43 \%$ of bladder tumors, the etiology of this tumor remains unknown. It is proposed that leiomyomas may arise from chromosomal abnormalities. ${ }^{1,2}$ Approximately $75 \%$ of the patients are young or middle aged. ${ }^{3}$ It is described as occurring at endovesical, extravesical, and intramural locations. $^{4}$ The tumor is usually asymptomatic unless urinary tract function is affected in symptomatic cases common presenting features are usually obstructive in nature. ${ }^{5}$ Behavior of leiomyoma of the bladder is by definition, completely benign. ${ }^{6}$

Treatment is delivered according to their size and anatomical location. ${ }^{7}$

Objective: A case of leiomyoma is presented because of confusing findings on imaging study (U/S and IVU).

\section{CASE REPORT}

A 24 years old married lady presented with recurrent attacks of irritative lower urinary tract symptoms and painless total hematuria twice over one month period.

The physical examination was unremarkable. Urinalysis revealed 3-5 RBCs/HPF and pus cells 35/HPF, uric acid crystals+ epith. cells+. Hemoglobin was $11.7 \mathrm{gm} / \mathrm{dL}$ and serum creatinine was $0.9 \mathrm{mg} / \mathrm{dL}$. Ultrasound study of the urinary tract revealed a $1.5 \times 2.5 \mathrm{~cm}$ solid SOL arising from the left posterior wall of the urinary bladder and upper tracts were normal. Figure 1.

IVU showed normal kidneys and ureter. Cystogram revealed filling defect in the bladder just close to the left ureteric orifice (confusing with a ureterocele before taking classical Cobra head feature when dye reachs lower ureter). Figure 2.

Cystoscopy.. revealed $1.5 \times 2.5 \mathrm{~cm}$ sessile mass in the posterior bladder wall just near the left 
ureteric orifice and was covered by normal bladder mucosa. Figure 3.

Trans Urethral Resection of Bladder Tumour (TURBT) was done with complete resection of the mass Figure 4.

Histopathological examination revealed leiomyoma of urinary bladder.

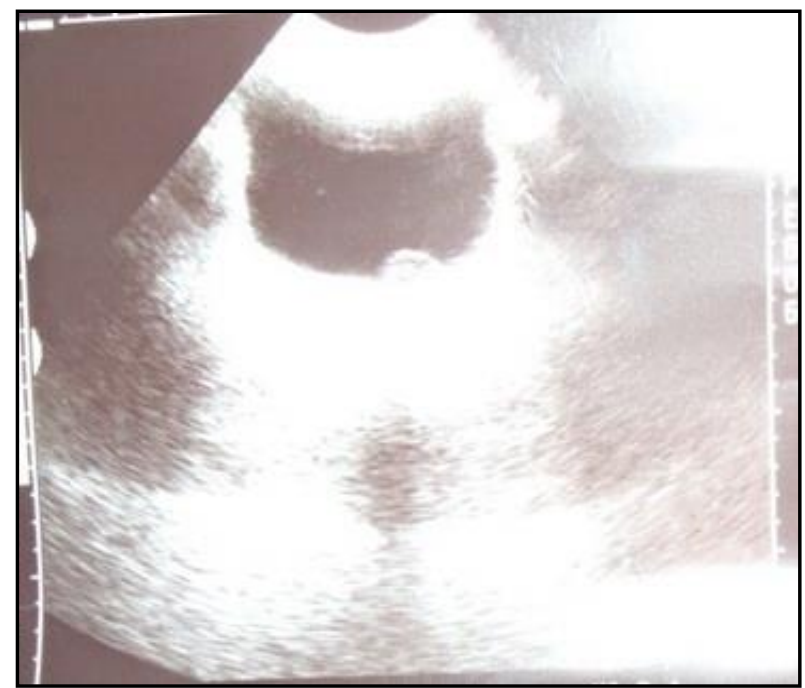

Figure 1. Ultra Sound..bladder mass.

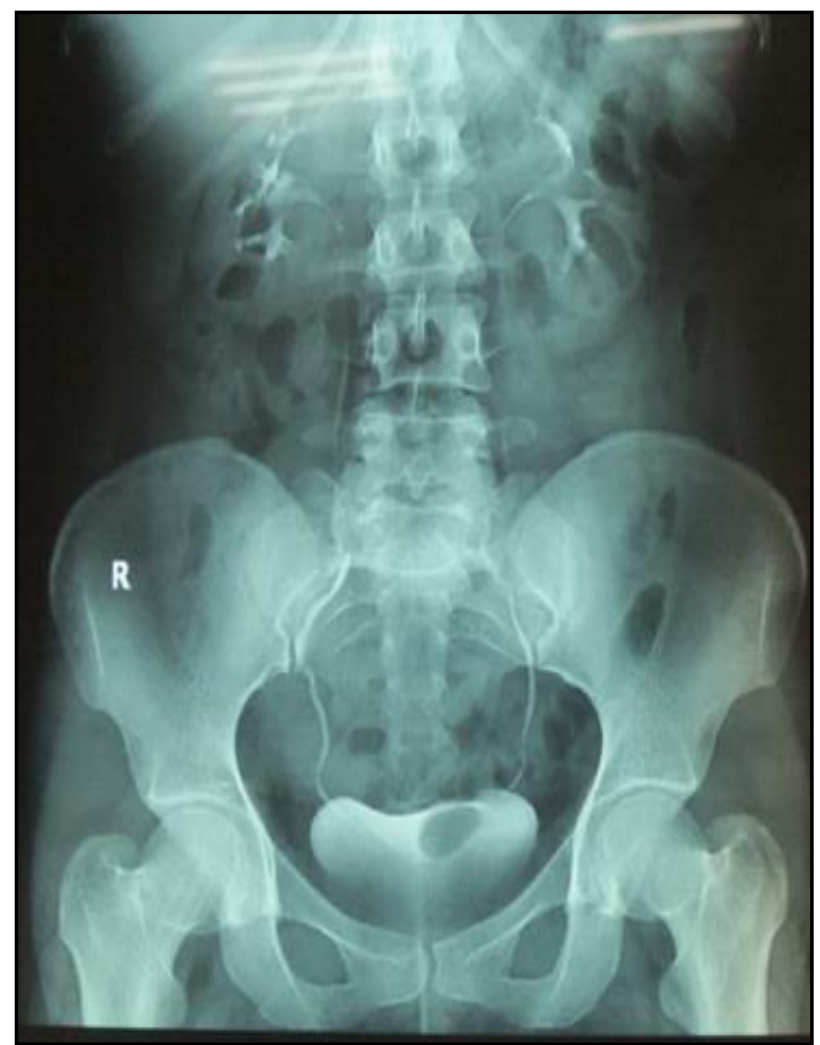

Figure 2. IVU..filling defect in the bladder.

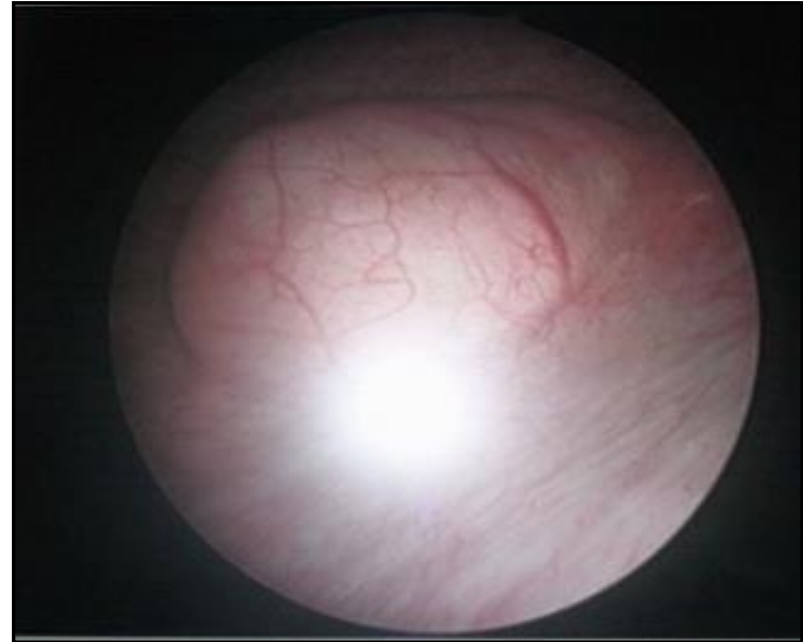

Figure 3. Cystoscopy..bladder mass.

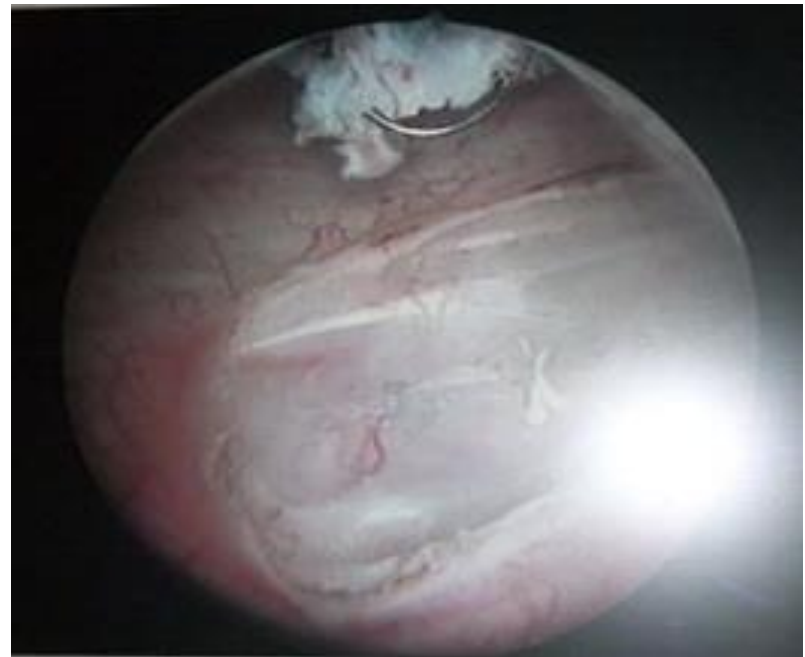

Figure 4. TUR-BT.

\section{DISCUSSION}

Most often, leiomyoma of the bladder remains asymptomatic unless its location obstructs the vesical outlet, causing urinary retention or urinary tract infection ${ }^{9}$. In this case irritative lower urinary tract symptoms were the presenting features without laboratory evidence of urinary tract infections. Clinical presentation differs from that of Chang and Chuang reported a 52-year-old postmenopausal woman presented with urinary urge incontinence of several months' duration ${ }^{5}$.

In Wong et al review, $62 \%$ of these patients were treated with open resection while $38 \%$ were treated with TURBT. ${ }^{8}$ TURBT was the method of treatment in this case

Ching-Yi et al reported a 53-year-old female presented with fever and right-side flank pain for a period of 1 month. (IVU) revealed right side 
hydronephrosis with a high-positioned bladder ${ }^{10}$ fever and hydronephrosis were not present in the current case, this is because of absence of obstructive effect of the tumour on the ureteric orifice.

A 68-year-old female of Caucasian origin presented with dysuria, urinary frequency and moderate abdominal discomfort. Cervical smears had been normal. An X-ray of the abdomen showed multiple calcifications within an ill defined mass extending out of the pelvis and she experienced menopause at the age of 52 reported by $\mathrm{G}$. Hudelist et $\mathrm{al}^{11}$ this differs from our reported case in the presence of calcification while tumour reported her devoid calcification probably due to early presentation.

Fistula between degenerated uterine leiomyoma and the bladder in a 57-year-old postmenopausal woman presented complaining of urinary incontinence, reported by Dmitry Fridman et al ${ }^{12}$ No such an association was found in the present case.

Sakellariou et al reported intramural leiomyoma in 28 years old female which was treated by partial cystectomy. $^{13}$

A 45-year-old woman presented with nearly 3year history of difficulty in passing urine in addition to frequency, urgency and intermittent incontinence of urine for the past 4 months, the left uretero-vesical junction was involved resulting in hydroureteronephrosis reported by Sudhakar et $a l^{14}$ it was treated by partial cystectomy, hysterectomy, and augmentation cystoplasty was performed with reimplantation of the left ureter due to large intraluminal mass (Leiomyoma) 10X8X7 $\mathrm{cm}$. compared to the present case which was much smaller in size not associated with hydronephrosis and was treated with TUR-BT only.

\section{CONCLUSION}

Leiomyoma should be kept in mind during differential diagnosis of filling defect in urinary bladder.

\section{REFERENCES}

1. Cornella JL, Larson TR, Lee R A, Marjina J F, Kammerer-Doak D. Leiomyoma of the female urethra and bladder: report of twenty-three patients and review of the literature. Am J Obst. Gyn. 1997 Jun 176(6):127885.

2. Nazir S, Maqbool A, Khan M. Leiomyoma of the Urinary Bladder-A Case Report and Brief Review of Literature. Int J Nephrol Urol, 2009; 1(1): 72 - 74.

3. Chen M, Lipson SA, Hricak H. MR imaging evaluation of benign mesenchymal tumors of the urinary bladder. AJR Am J Roentgenol 1997; 168: 399-403.

4. Kutzmann, A. A. Leiomyoma of the urinary bladder. J Urol 1937; 37: 117-32.

5. Chang $P$, Chuang $C$. Bladder Leiomyoma Presenting as Urinary Incontinence: A Case Report. JTUA 2009; 20(4):178-80.

6. Subudhi C L, Panda S, Pradhan D, Pati N. Leiomyoma of the urinary bladder. Indian J Urol. 2002 Jun 18:166-8.

7. Soloway D, Simon MA, Milikowski C,Soloway MS. Epithelioid leiomyoma of the bladder: an unusual cause of voiding symptoms. Urology. 1998 Jun;51(6):1037-9.

8. Wong FK, Wong YFM, Ma KF, Lui CY. Urinary bladder leiomyoma. J HK Coll Radiol. 2002; 5: 193-6.

9. Khaitan A, Seth A, Dinda A K, Singh I, Talwar M \& Bandhu S. Transurethral Resection versus Open Surgery for Leiomyoma of Urinary Bladder - A Report of 2 Cases. Int Urogynecol J (2002) 13:270-273.

10. Ching-Yi L, Wang L, Chuang K, Kwai-Fong Wu , Chuang C. Bladder leiomyoma with right hydronephrosis. Int Urogynecol J 2006; 27: 215-217.

11. Hudelist G, Fouad A, English J, Liton T, Beard R. Calcifying uterine leiomyoma invading the urinary bladder. Gynecol Surg 2007 june 1: 295-297.

12. Fridman D, Chakraborty S, Khulpateea N. Fistula between degenerated uterine leiomyoma and the bladder: case report. Int Urogynecol J 2011 March 31 22:1329-1331.

13. Sakellariou $P$, Protopapas A, Kyritsis N, Voulgaris Z, Papaspirou E, Diakomanolis E. Intramural Leiomyoma of urinary bladder. Eur. Radiol. (2000).10. 906-908.

14. Sudhakar P J, Malik N , Malik A. Leiomyoma of Bladder. Saudi J Kidney Dis Transpl 2008; 19: 232-5. 